\title{
MULTICAST ROUTING IN ATM NETWORKS
}

\author{
Chi-Chung Cheung ${ }^{1}$, Hon-Wai $\mathrm{Chu}^{2}$, Danny H.K. Tsang ${ }^{3}$ and Sanjay Gupta ${ }^{4}$ \\ ${ }^{1}$ Department of Computer Science \\ ${ }^{2}$ School of Science and Technology \\ City University of Hong Kong \\ csccc@cityu.edu.hk \\ Open University of Hong Kong \\ ${ }^{3}$ Department of Electrical and \\ Electronic Engineering \\ Hong Kong University of \\ wchu@ouhk.edu.hk \\ Science and Technology \\ eetsang@ee.ust.hk \\ ${ }^{4}$ GSM Products Division \\ Motorola, USA \\ guptasn@cig.mot.com
}

\begin{abstract}
In this paper, we study the problem of multicast routing in ATM based networks. We propose a dynamic multicast algorithm in ATM networks called Least Load Multicast Routing (LLMR) algorithm and the algorithm with statistical multiplexing is considered. A numerical example is presented to show the characteristics of LLMR. Moreover, we find that the connection blocking probabilities decrease with increasing destination size.
\end{abstract}

Keywords: ATM Networks, Multicast Routing.

\section{INTRODUCTION}

Multicasting refers to the ability of a set of more than two nodes or endusers in a communication network to communicate simultaneously with each other. Applications that require multicast capability (either point-to-multipoint (PTM) as in distributional video or multipoint-to-multipoint (MTM) as in video conferencing, online collaboration and others) will be an integral part of future broadband services. Asynchronous Transfer Mode (ATM) has been almost universally accepted as the multiplexing and switching technique for Broadband Integrated Services Digital Networks (B-ISDN). ATM - a high-speed, Virtual Circuit (VC) oriented packet-switching technique that uses fixed length packets called cells - provides an acceptable and cost effective means for meeting the requirements of future broadband networks [8]. 
Given the popularity of ATM based broadband networks we study the problem of multicast routing in ATM based networks. Recent research efforts in the area of multicast routing (few of them have been conducted keeping in perspective the requirements of ATM networks) have followed the following broad approach: the underlying network is modeled as a graph (directed or undirected) with links as edges and switches as nodes; and each edge is assigned a "cost" (for instance the cost could be a measure of expected delay). The multicast routing problem is then reduced to finding a "tree" that spans the nodes that wish to participate in a given multicast connection that has minimal cost [1][4][7][10][11][12]. The above problem is known in the literature as the Steiner tree problem (an NP-complete [9] problem) for which several heuristics have been proposed. Recently, [3] proposed dynamic algorithms to handle the multicast routing problem in circuit switching networks and the bandwidth of each connection is peak rate allocated. In this paper, we proposed a dynamic multicast algorithms in ATM networks called Least Load Multicast Routing (LLMR) algorithm.

In this paper we make a distinction between ATM networks operating in the statistical and non-statistical multiplexing modes. An ATM network operates in the statistical multiplexing mode if, for any VP, the VCs routed over the VP are permitted to have an aggregate offered bit rate that exceeds the bandwidth allocation of the VP. During these excess periods, the buffer before the first link of the VP will accumulate with cells and perhaps overflow. The ATM network is operated in the non-statistical multiplexing mode if, for each VP, the aggregate instantaneous bit rate offered by the VCs does not exceed the VP's bandwidth allocation ${ }^{1}$.

In ATM based networks there is also a distinction between point-to-multipoint (PTM) and multipoint-to-multipoint (MTM) VCs. In a PTM VC, there exists a single source (henceforth referred to as root) and a set of destination nodes, and information is sent from the source to the destinations. We would like to emphasize that no information is sent from the destinations to the source or other destinations. On the other hand in an MTM VC all participating nodes communicate with each other.

The paper is organized as follows. We describe the relevant features of ATM networks and their architecture briefly in Section 2. In Section 3 we propose multicast routing policies for various classes of ATM networks. In Section 4 we present the simulation results and compare it with other dynamic multicast routing algorithms. Finally, we conclude in Section 5 by summarizing the paper and outlining avenues for future research. 


\section{MULTICAST ROUTING POLICIES}

Consider the VP network to be a graph $G=(V, E)$ where $V$ and $E$ are the set of nodes and VPs respectively. Denote by $C(e), e \in E$, the bandwidth allocated to VP $e$. The routing policies that we propose in the paper is employed for routing PTM VC connection requests and thus the underlying graph is assumed to be directed. For routing MTM VC connection requests, since ATM supports PTM VC connections with asymmetric bandwidth, we can employ multi PTM VC connections to support MTM VC connection requests. Each node in a group that wishes to communicate can establish a PTM VC connection to all of the other nodes in the group. For a group of N nodes, this requires N PTM $\mathrm{VC}$ connections.

We consider the case where statistical multiplexing is performed within VP subnetworks. For reasons of notational simplicity, we consider a case of homogeneous VCs, (i.e., all VCs have identical traffic characteristics and QOS requirements). In order to simplify the discussion, we assume that the QOS requirement only involves cell loss. Specifically, for each VC in progress, suppose that the fraction of cells lost are not permitted to exceed $\epsilon$ (typical values of $\epsilon$ are between $10^{-6}$ and $\left.10^{-9}\right)$. Let $p_{e}(l)$ be the fraction of cells lost at the input buffer of VP $e$ when $l$ VCs are being routed through VP $e$, these probabilities can be determined by a cell-level analysis; for example, see [2][ 5][13].

Consider an VC request with destination set $S(c)$ and a connected, acyclic graph (tree) $T=(V(c), E(c)), S(c) \subseteq V(c)$. Nodes $s \in V(c)-S(c)$ are referred to as alternate nodes. A VP, $e \in E(c)$, is referred to as an alternate $\mathrm{VP}$ if it connects to an alternate node. Let $t(e)$ denote the reservation threshold for $\mathrm{VP} e$, i.e., $\mathrm{VP} e$ can be used as an alternate VP only if its residual capacity exceeds $t(e)$. Let $\pi^{T}\left(s_{1}, s_{2}\right), s_{1}, s_{2} \in V(c)$ be the set of VPs in the path between the nodes $s_{1}, s_{2}$. The tree $T$ can be used for routing VC request $c$, if and only if

(a) the QOS requirement of the VC being routed is satisfied, i.e.,

$$
\prod_{e \in \pi^{T}\left(s_{1}, s_{2}\right)}\left[1-p_{e}\left(l_{e}+1\right)\right]>1-\epsilon \text { for all } s_{1}, s_{2} \in S(c)
$$

(we have implicitly assumed that the cell loss probabilities on distinct VPs are independent) and

(b) the QOS requirement of any pre-existing $\mathrm{VC}$ is not violated after addition of VC $c$ on tree $T$. (At the very least, all existing VCs that are routed through $\mathrm{VP} e, e \in E(c)$ will have to be (re)examined.)

Checking for condition (b) above is computationally prohibitive, even for small networks, as it involves examining VPs that are NOT in $V(c)$. It can be 
demonstrated that for a large fraction of $\mathrm{VC}$ requests, before a multicast $\mathrm{VC}$ can be assigned a routing tree, the status of all the VPs in the VP subnetwork will be required. (For a detailed discussion refer to [6].) We would like to remind that QOS requirements often involve bounds on delay as well. In this case step (a) and step (b) will involve convolution of random variables - a computationally intensive exercise. Further, the overhead, due to the increased information exchange required, on the signaling network, increases substantially. In light of the above discussion, it is easy to see that determining the acceptability of an $\mathrm{VC}$ request on any given tree places significant stress on the network management and control protocols and is therefore unacceptable.

In order to reduce the computational requirements placed on the switches and the burden on network management and control functions, it is critical that a VP be able to decide if it can be used as a part of the routing tree without requesting any information from other VPs in the VP subnetwork or the tree. Therefore we propose the following (more restrictive) admission rules for determining if a $\mathrm{VC}$ request $c$ can be routed on a tree $T=(V(c), E(c)), S(c) \subseteq V(c)$ :

\section{Revised Admission Rules}

Rule $1 p_{e}\left(l_{e}+1\right)<\epsilon_{e}^{*}, \epsilon_{e}^{*} \leq \epsilon$ for all $e \in E(c)$, have been chosen a priori; and

Rule $2 \prod_{e \in \pi^{T}\left(s_{1}, s_{2}\right)}\left[1-\epsilon_{e}^{*}\right]>1-\epsilon$ for all $s_{1}, s_{2} \in S(c)$.

When operating under the scope of the revised admission rules, the admissibility of a $\mathrm{VC}$ on any routing tree can be determined after considering the state of VPs that are a part of the routing tree only, thus reducing the complexity of the admissibility criterion. However, the above simplicity in admissibility criterion is achieved at the expense of denying VC requests when there exist feasible trees (possibly minimal) to route them.

The revised admission rules transform (for purposes of routing VCs only) a VP subnetwork with statistical multiplexing to an equivalent VP subnetwork with no statistical multiplexing. Note that in this case the capacity of VP $e$ is defined as

$$
C(e):=\max \left\{l: p_{e}\left(l_{e}\right) \leq \epsilon^{*}\right\} .
$$

The revised admission rules require that in networks with statistical multiplexing the diameter of the routing trees not exceed $\epsilon / \epsilon^{*}$. Complexity of algorithms that construct maximum residual capacity trees with constraints on their maximum diameter are substantial. Therefore, we propose that routing policies that limit the maximum number of hops from a given node to others in the destination (henceforth referred to as the distance between destination nodes) set be employed. For instance, if the distance from the source node to any other destination node can not exceed $h^{*}$, the maximum diameter of the tree 
will certainly be less than $2 h^{*}$. Hence, for MTM VCs we propose using $h^{*}$ - the maximum distance between the source and any other destination node - to be $\frac{\epsilon}{2 \epsilon^{*}}$; however, for PTM VCs where data flows from one node to the remaining nodes in the destination set choosing $h^{*}=\frac{\epsilon}{\epsilon^{*}}$ is clearly sufficient. We now propose the following routing policy for ATM networks with statistical multiplexing for a $\mathrm{VC}$ request with destination set $V(d)$, source node $s_{r}$ and a maximum distance of $h^{*}$ from the source node to any other destination node:

\section{Least Loaded Multicast Routing (LLMR)}

1. Initialization: Let $T_{1}=\left(V_{1}+V_{t}, E_{1}\right)$ where $V_{1}=s_{r}, V_{t}=\phi$, and $E_{1}=\phi$. Set $i=1$ and proceed to Step 2 (VP selection).

2. VP Selection: If $S(c) \subseteq V_{i}+V_{t}$ the route selection process is complete and the tree $T=\left(V_{i}+V_{t}, E_{i}\right)$ is used to route the VC request. If $i<d(c)$ choose VP $e^{*}=\left(s_{1}^{*}, s_{2}^{*}\right), s_{1}^{*} \in V_{i}+V_{t}, s_{2}^{*} \in S(c)-V_{i}-V_{t}$, such that $R\left(e^{*}\right) \geq R(e)$ where $e=\left(s_{1}, s_{2}\right)$ is a VP such that $s_{1} \in V_{i}+V_{t}, s_{2} \in$ $S(c)-V_{i}-V_{t}$, (any ties are broken randomly). If $R\left(e^{*}\right) \geq b(c)$ and the distance between $s_{r}$ and $s_{2}^{*}$ is $\leq h^{*}$ then (i) $E_{i+1}=E_{i}+e^{*}$ and $V_{i+1}=V_{i}+s_{2}^{*}$ and (ii) set $i=i+1$ and Step 2 is repeated (VP selection); Otherwise search for an alternate node is initiated as in Step 3.

3. Choose an alternate node: If $V_{t} \neq \phi$ the VC request is denied; otherwise (if $V_{t}=\phi$ ) define an alternate path, $\psi$, as the set of VPs $e_{1}=\left(s_{1}, s_{3}\right), e_{2}=\left(s_{3}, s_{2}\right)$ such that (i) $s_{1} \in V_{i}, s_{2} \in S(c)-V_{i}$, (ii) $s_{3} \in V-S(c)$, and (iii) distance between $s_{r}$ and $s_{2}$ is $\leq h^{*}$. Let $R(\psi)=\min \left\{R\left(e_{1}\right)-t\left(e_{1}\right), R\left(e_{2}\right)-t\left(e_{2}\right)\right\}$ be the residual capacity of the alternate path $\psi$. Denote by $\psi^{*}$ the alternate path with maximum residual capacity. If $R\left(\psi^{*}\right) \geq b(c)$ then (i) $V_{t}=\left\{s_{3}^{*}\right\}$; (ii) $V_{i+1}=V_{i}+s_{2}^{*}$; and $E_{i+1}=E_{i}+e_{1}^{*}+e_{2}^{*}$; (ii) set $i=i+1$ and (iii) proceed to Step 2 (VP selection). If $R\left(\psi^{*}\right)<b(c)$, the $V C$ request is denied.

Observe that in LLMR a minimal tree with the largest residual capacity is chosen for routing the VC. In case no minimal tree are available, from the set of trees with no more than one alternate nodes the tree having the largest residual capacity is chosen. In case no such tree exists with the required bandwidth available on each component VP, the VC request is denied. Note that we only allow at most one alternate node added to establish any given $\mathrm{VC}$ request. It is because there is only marginal improvement in performance when we use more than one alternate node (further if the load on the network is sufficiently high the performance of the system degrades by using increasing number of alternate nodes). We will show this fact through the simulation result in Section 3.

To illustrate the routing algorithm outlined above consider a PTM VC request with the destination set $\{2,3,4\}$ and source node 1 . The diameter of the routing 
tree is not allowed to exceed 3. The steps in the connection establishment procedure are as shown in Figure 1 - the numbers next to a VP denote its residual capacity and VPs that are included in the set $E(c)$ are drawn with "thick" lines. alternate paths and alternate nodes are not shown for clarity. Note that node 3 is the first node to be added as the VP $(1,3)$ has the largest residual capacity (3) among VPs, $(1,4)$ and $(1,2)$, that are connected to node 1 - the root node. Subsequently, nodes 2 and 3 (in this order) are added to the routing tree and the VPs included are $(3,2)$ and $(2,4)$, respectively.

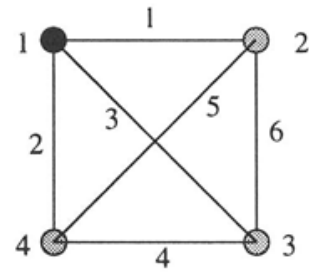

Iteration 1

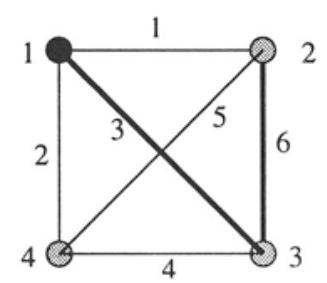

Iteration 3

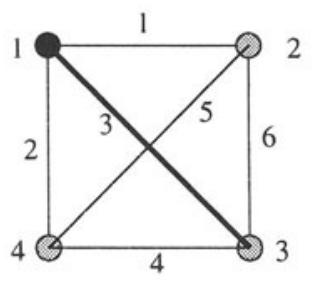

Iteration 2

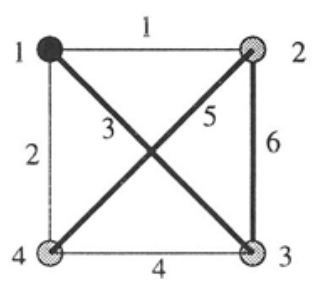

Iteration 4
Root node

Destination node

Figure 1 Example to illustrate LLMR without alternate node

Figures 2 and 3 consider a 6-node network and a $\mathrm{VC}$ request with destination set $\{1,3,5\}$ with node 6 as the source node. In figure $2, \epsilon^{*}$ is chosen such that the diameter of the routing tree is not allowed to exceed 4 . Node 3 is the first one to be added as VP $(6,3)$ has a residual capacity of 6 that is larger than the residual capacities of the VPs $(6,1)$ and $(6,5)$ which is followed by node 5 (VP $(6,5)$ is chosen due to its larger residual capacity over VP $(3,5)$ which has a residual capacity of 0 ). Search for a VP to node 1 from the nodes 3,5 , and 6 reveals that there are no available VPs with non-zero residual capacities and therefore the search for an alternate node is initiated. For the case at hand, node 2 is selected as the alternate node (the alternate path chosen is shown by dashed lines). In figure 3 , the diameter of the routing tree is limited to be not greater than 3. The connection establishment procedures of destination nodes 3 and 5 are the same as the example in Figure 2. However, the alternate path selected for node 1 is different to the previous example since the diameter of the routing tree of the previous example is 4 , which is not allowed in this case. 
Thus the second best is selected and the diameter of the routing tree is limited not to exceed 3.
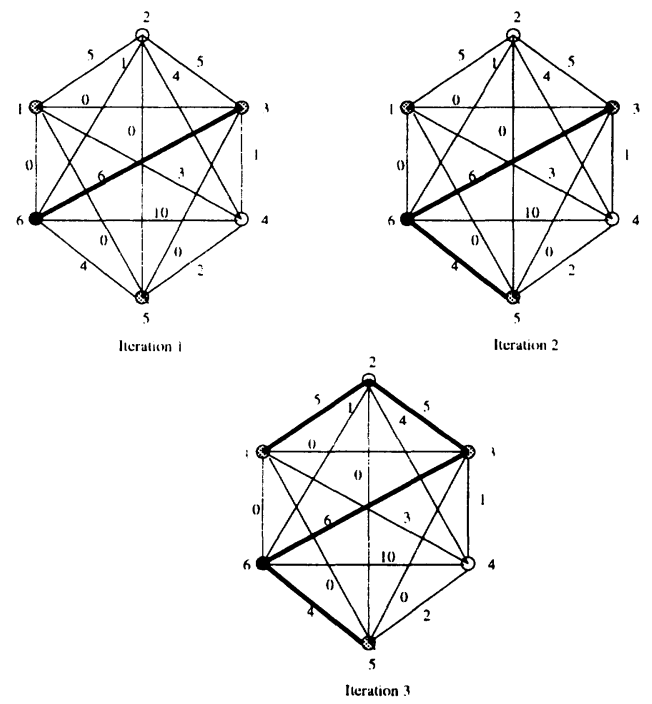

- R(x)t moxk

- Destination node

Other nodex

Figure 2 Example to illustrate LLMR with an alternate node
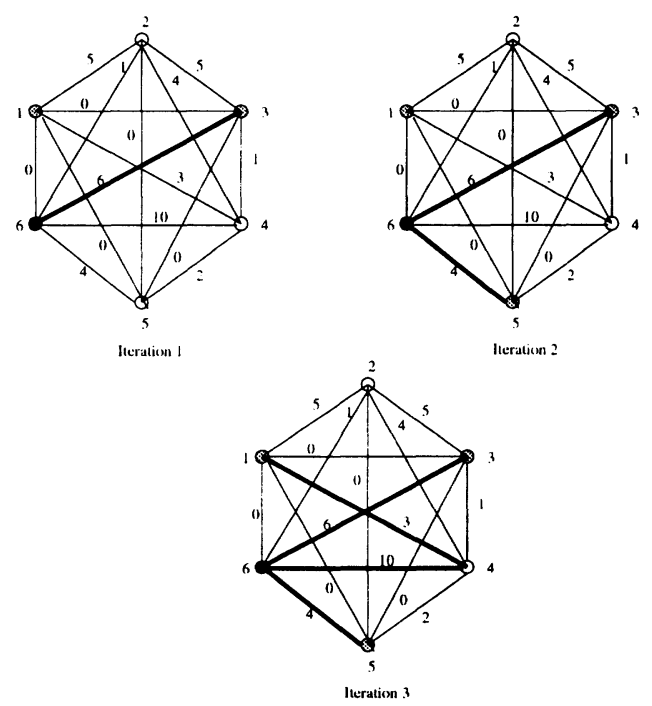

- R(x)t nole

- Destination node

Other nodes

Figure 3 Example to illustrate LLMR with an alternate node 
We now determine the complexity of the LLMR routing algorithm. When no alternate nodes are employed the number of VPs queried are $d(d-1) / 2$ and the total number of comparisons needed is equal to that required in sorting $d(d-1) / 2$ numbers which is $O\left(d(d-1) \log _{2} d\right)$. The time complexity of the above is $d-1$, i.e., the time required to set-up a $\mathrm{VC}$ request with a destination set of size $d$ is $d-1$ times the time required to set-up a point-to-point VC request. The use of an alternate node increases the time complexity to $d$. The number of VPs whose status is requested depends on which intermediate stage the alternate node is added. If the alternate node is added at stage $i$, i.e., when there are $i$ destination nodes in the routing tree constructed thus far, the number of VPs queried for their status are $i(d-i)$ direct VPs and $2 i(d-i)(N-d)$ alternate VPs. In the worst case the number of VPs whose status is requested is less than $d(d-1) / 2+\frac{d^{2}}{2}(N-d)$.

\section{SIMULATION RESULTS}

We consider a fully connected network with 12 nodes and each link have a capacity of 30 bandwidth units. The maximum destination size of a connection request is 6 . In order to maintain the same link load of each type of VC requests, the arrival rate, $\lambda_{d}$, of a $\mathrm{VC}$ request with destination size $d$ is assumed to be inversely proportional to the minimum number of links, $d-1$, used to establish the VC, i.e. $(d-1) \lambda_{d}=\lambda$, where $\lambda$ is a constant. For convenience we define the normalized network load, $L$, as the ratio of total offered load to total network capacity, i.e.,

$$
L=\frac{\sum_{d=2}^{6} L_{d}}{\sum_{e \in E} C(e)} .
$$

For each set of simulation point, 10 independent runs are carried out and the length of each run is $10^{6}$ units of mean VC request interarrival time. For each run, the initial $10 \%$ of each run was discarded to avoid the effect of transient state. The symbols around each simulation point are to show the upper and lower bounds of $95 \%$ confidence interval.

The performance of LLMR algorithm is shown in Figure 4. When the loading of a network is high (larger than 100\%), the normalized revenue losses is also high since the network resources are insufficient and no algorithm can help under this situation. However, when the loading is moderate, the normalized revenue loss of LLMR algorithm decreases rapidly because LLMR select least loading path to avoid congestion. Figure 5 shows that the normalized revenue loss with at most alternate node is much smaller than that without any alternate node. Furthermore, the revenue loss changes only marginally when the maximum number of alternate nodes allowed per $\mathrm{VC}$ request increases. It is because more resources are used to make connection over alternate paths and 
less resources are for future $\mathrm{VC}$ requests. It is therefore prudent to restrict the maximum number of alternate nodes to at most one for multicast routing.

Figure 6 shows an interesting phenomenon: the larger the destination set size of the $\mathrm{VC}$ request the smaller the blocking probabilities. It is because there are two factors affecting the $\mathrm{VC}$ blocking probability: the number of links required and the number of possible choices to establish a multicast VC. A VC request with a large destination set requires more links so that its blocking probability should be higher than that with smaller destination set. However, the number of possible links to build a connected tree is also larger and thus there is higher chance to avoid congestion and blocking. For example, in a fully connected network, there are 3 choices to establish a connected tree if the destination size is 3 , but for a destination size of 4,16 choices are available to establish a VC. That means a VC request for a destination size of 4 has 13 more choices to successfully establish than that for a destination size of 3 . It turns out the latter factor dominates in a VC request with larger destination set.

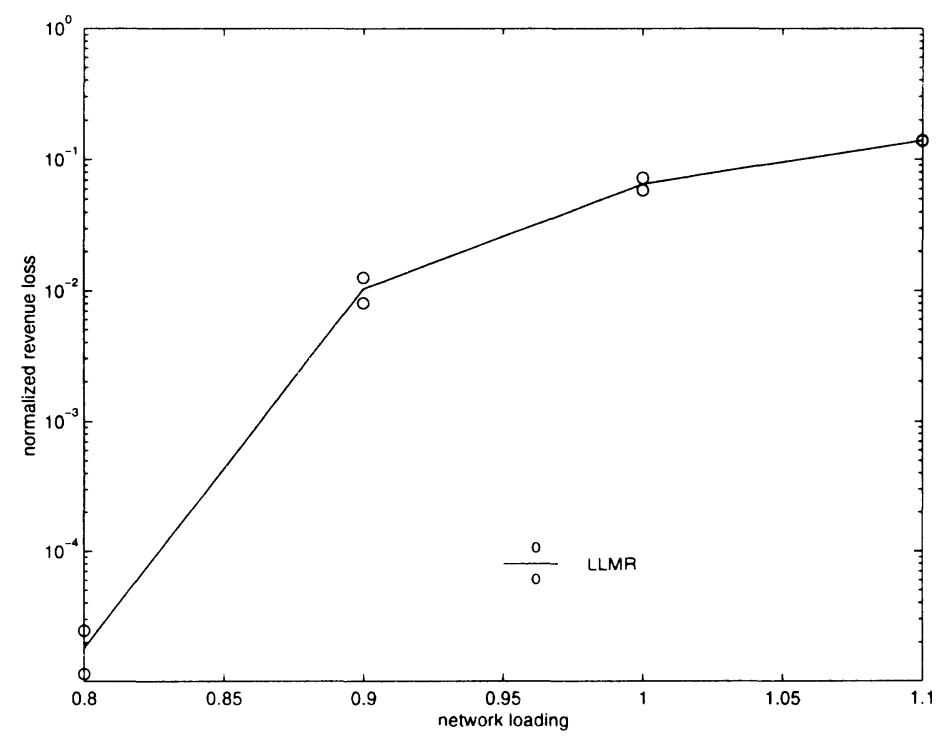

Figure 4 Normalized revenue loss vs. network loading 


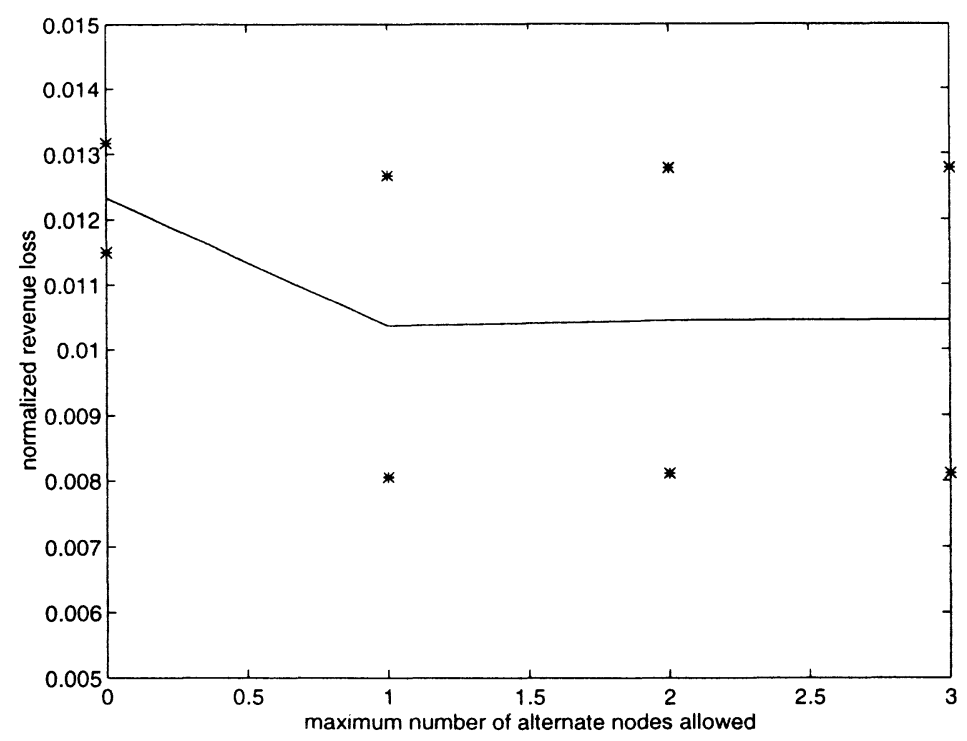

Figure 5 Normalized revenue loss vs. maximum number of alternate nodes allowed (offered load $=0.9$ )

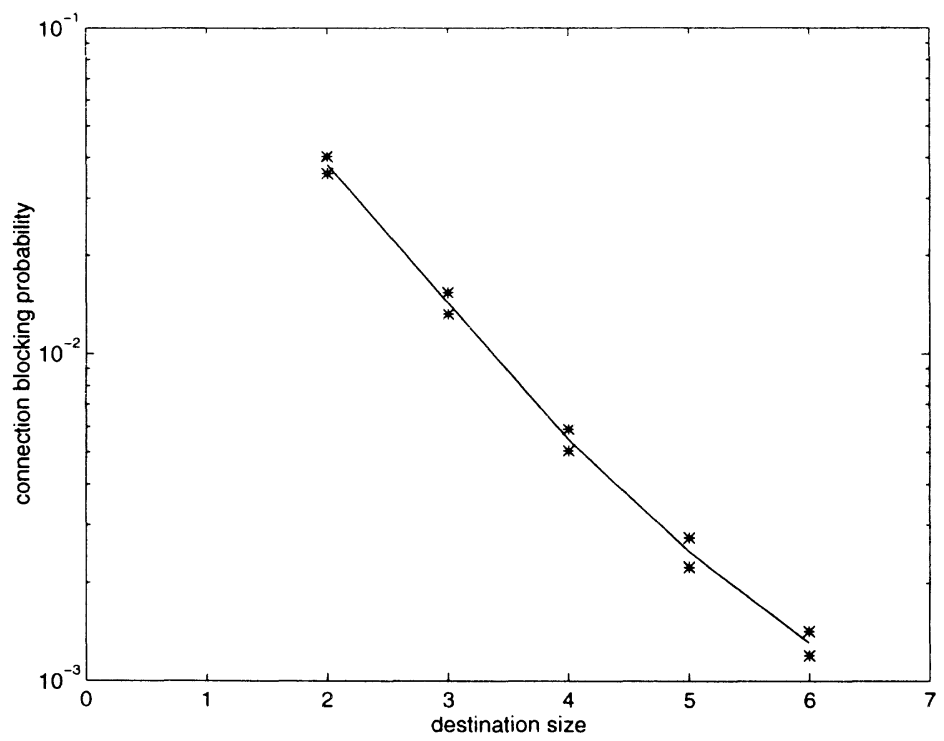

Figure 6 VC blocking probability vs. destination size (offered load=0.9) 


\section{CONCLUSIONS}

In this paper, we propose new dynamic multicast routing algorithms for ATM networks and the algorithms with statistical multiplexing are considered. The simulations results shows the characteristics of LLMR. Moreover, we find that the connection blocking probabilities decrease with increasing destination size.

\section{Notes}

1. The wording non-statistical multiplexing has been chosen for consistency with the terminology in the ATM literature. We stress, however, that even for non-statistical multiplexing the cells propagating on a VP are "randomly" allocated to the various VCs established over the VP.

\section{References}

[1] M. H. Ammar, S. Y. Cheung, and C. M. Scoglio. Routing multipoint connections using virtual paths in an ATM network. In Proceedings of INFOCOM, pages 98-105, 1993.

[2] D. Anick, D. Mitra, and M. M. Sondhi. Stochastic theory of a datahandling system with multiple sources. The Bell Systems Technical Journal, vol. 61:1871-1894, 1982.

[3] Chi-Chung Cheung, D.H.K. Tsang, S. Gupta and H-W Chu. Least loaded first multicast routing in single rate loss networks. To appear in ICC '96, 1996.

[4] M. Doar and I. Leslie. How bad is naive multicast routing? In Proceedings of INFOCOM, pages 82-89, 1993.

[5] R. Guerin, H. Ahmadi, and M. Naghshineh. Equivalent capacity and its application to bandwidth allocation in high-speed networks. IEEE Journal on Selected Areas in Communications, vol. 9:968-981, 1991.

[6] S. Gupta, K. W. Ross, and M. El Zarki. On Routing in ATM Networks. Prentice-Hall and Manning, 1995. Routing in Communications Networks.

[7] V. P. Kompella, J. C. Pasquale, and G. C. Polyzos. Multicasting for multimedia communication. In Infocom, pages 2078-2085, 1992.

[8] J. B. Lyles and D. C. Swinehart. The emerging gigabit environment and the role of local ATM. IEEE Communications Magazine, vol. 30:52-58, April 1992.

[9] M. Minoux. A class of combinatorial problems with polynomially solvable large scale set covering/partitioning relaxations. R.A.I.R.O Operations Research, vol. 21(2):105-134, 1988. 
[10] H. Tode, Y. Sakai, M. Yamamoto, H. Okada, and Y. Tezuka. Multicast routing algorithm for nodal load balancing. In Infocom, pages 2086-2095, 1992.

[11] B. M. Waxman. Routing of multipoint connections. IEEE Journal on Selected Areas in Communications, vol. 6:1617-1622, December 1988.

[12] B. M. Waxman. Performance evaluation of multipoint routing algorithms. In Infocom, pages 980-986, 1993.

[13] T. Yang and D.H.K. Tsang. A novel approach to estimating the cell loss probability in an ATM multiplexer loaded with homogeneous on-off sources. IEEE Transactions on Communications, vol. 43:117-126, Jan. 1995. 